\title{
Gamificación en contextos educativos: análisis de aplicación en un programa de contaduría pública a distancia*
}

\section{Jennifer Lorena Gómez Contreras**}

Fecha de recibido: 28 de junio de 2018

Fecha de aprobado: 21 de junio de 2019

Para citar: Gómez Contreras, J. L. (2020). Gamificación en contextos educativos: análisis de aplicación en un programa de contaduría pública a distancia. Revista Universidad \& Empresa, 22(38), 8-39. https://doi.org/10.12804/revistas.urosario.edu.co/ empresa/a.6939

* Resultado del proyecto INV-DIS-2573 titulado Evaluación de la influencia del modelo pedagógico institucional sobre las estrategias didácticas adoptadas por los docentes del programa de Contaduría Pública a Distancia de la Universidad Militar Nueva Granada (Sede Cajicá) financiado por la Vicerrectoría de Investigaciones de la Universidad Militar Nueva Granada, vigencia 2018

** Magíster en Administración y Contadora Pública de la Universidad Nacional de Colombia, sede Bogotá. Docente de planta tiempo completo, Programa de Contaduría Pública a Distancia, Universidad Militar Nueva Granada, sede Cajicá. Miembro del grupo de investigación INCONDIs. Correo electrónico: jennifer.gomez@unimilitar.edu.co 


\section{Resumen}

La escasez de estudios en donde se vincule la aplicación de gamificación educativa, con sus fundamentos teóricos y/o principios, ha llevado al fracaso de la gamificación en contextos educativos. En el presente artículo de reflexión se busca contribuir a solventar esta falencia conectando la aplicación de la gamificación educativa realizada en el programa de Contaduría Pública a Distancia de la Universidad Militar Nueva Granada (Colombia), con los fundamentos teóricos del tema. Para lo cual, se abordan las diferencias existentes entre la gamificación y el aprendizaje basado en juegos, seguidamente se examina el concepto, los objetivos, los aportes y los elementos de la gamificación, posteriormente se identifican los aspectos clave para una implementación exitosa en el contexto educativo. Finalmente, se explica el proceso de gamificación implementado, desde la planeación docente hasta el análisis de las opiniones de los estudiantes. Para ello, se utilizó una metodología exploratoria soportada en análisis documental y aplicación de encuestas a estudiantes. Como resultado de la aplicación realizada se verificó que la gamificación educativa promueve la autonomía, la motivación, el compromiso y la interacción social en los estudiantes, permitiendo a su vez la progresión y el dominio de los temas abordados en cada reto propuesto.

Palabras clave: gamificación, aprendizaje, motivación, contexto educativo, educación contable a distancia.

\section{Gamification in Educational Contexts: Analysis of Its Application in a Distance Public Accounting Program}

\section{Abstract}

The scarcity of studies that link the application of educational gamification to its theoretical foundations or principles has led to the failure of gamification in educational contexts. This paper seeks to contribute to solving this shortcoming by connecting the application of the educational gamification carried out in the Distance Public Accounting Program of the Universidad Militar Nueva Granada (Colombia) with the theoretical foundations of the subject. To this end, researchers addressed the differences between gamification and game-based learning, examined the concept, objectives, contributions, and elements of the gamification, and identified the key aspects for a successful implementation in the educational context. Finally, they explained the process of gamification implemented from the teacher planning to the analysis of student opinions. For this, the methodology used was an exploratory one supported in documentary analysis and application of surveys to students. As a result of the application, researchers verified that educational gamification promotes autonomy, motivation, commitment, and social interaction in the students, allowing the progression and mastery of the issues covered in each proposed challenge.

Keywords: Gamification, learning, motivation, educational context, distance accounting education.

\section{Gamificação em contextos educativos: análise de aplicação em um programa de contadoria pública a distância}

\section{Resumo}

A escassez de estudos onde se vincule a aplicação de gamificação educativa com seus fundamentos teóricos e/ou princípios, tem levado ao fracasso da gamificação em contextos educativos, no presente artigo de reflexão se busca contribuir a resolver esta falência conectando a aplicação da gamificação educativa realizada no programa de Contadoria Pública a Distância da Universidad Militar Nueva Granada (Colômbia) com os fundamentos teóricos do tema. Para isto, abordam-se as diferenças existentes entre a gamificação e a aprendizagem baseada em jogos, seguidamente examina-se o conceito, os objetivos, os aportes e os elementos da gamificação, posteriormente se identificam os aspectos chave para uma implementação de sucesso no contexto educativo. Finalmente, se explica o processo de gamificação implementado, desde o planeamento docente até a análise das opiniões dos estudantes. Para isto, se utilizou uma metodologia exploratória suportada em análise documental e aplicação de enquetes a estudantes, como resultado da aplicação realizada se verificou que a gamificação educativa promove a autonomia, a motivação, o compromisso e a interação social nos estudantes, permitindo ao mesmo tempo a progressão e o domínio dos temas abordados em cada desafio proposto.

Palavras-chave: gamificação, aprendizagem, motivação, contexto educativo, educação contável a distância. 


\section{Introducción}

Un problema importante que muchas escuelas y educadores enfrentan hoy en día es que muchos estudiantes carecen de la motivación y el interés para aprender. Más aún, si se les da la opción, muchos de ellos preferirían jugar videojuegos en lugar de leer un libro o completar una tarea (Zichermann \& Cunningham, 2011). Teniendo en cuenta este escenario, han surgido diferentes innovaciones pedagógicas que incorporan la lógica de los juegos, tales como la gamificación y el aprendizaje basado en juegos (ABJ).

Existen diferentes estudios que han abordado los aspectos relacionados con la gamificación y el ABJ, de la revisión de literatura realizada se identifican cinco tendencias principales de investigación, en las cuales se agrupan los estudios realizados sobre estas temáticas, estas son:

- Impacto de la gamificación en la actitud, la motivación y el compromiso de los estudiantes: en esta tendencia se encuentran estudios que abordan cómo los juegos serios impactan la actitud, la motivación, la innovación y el compromiso de los estudiantes (Ahmed \& Sutton, 2017), los elementos de los videojuegos que se utilizan en el contexto pedagógico para promover el compromiso, la motivación y las conductas deseadas de los estudiantes (Alsawaier, 2018), la influencia de la gamificación en aspectos motivacionales, cognitivos y sociales (Deif, 2017), el efecto de la gamificación en el compromiso de los estudiantes (Lim, Nonis \& Hedberg, 2006; Spires Rowe, Mott \& Lester, 2011), cómo el uso de la gamificación educativa impacta en la actitud, la motivación y el interés de los estudiantes (Ting, 2010; Li, 2010), y el efecto de los elementos del juego en la motivación y la participación de los usuarios (Zeng, Tang \& Wang, 2017).

- Aplicación de la gamificación educativa y del ABj: hacen parte de esta agrupación los trabajos encaminados a analizar el concepto de gamificación y su aplicación en la enseñanza de diversas áreas de conocimiento (Barab, Zuiker, Warren, Hickey, Ingram-Goble, Kwon \& Herring,2007; Gåsland, 2011; Antin, 2009; Hsu, Tsai \& Liang, 2011), el uso de juegos y simulaciones en el aprendizaje (Burgess, 1991; Wolfe, 1993; Faria, 1987; Faria 1998, Faria \& Wellington, 2004), el aprendizaje basado en juegos en la educación cientí- 
fica (Li \& Tsai, 2013), el uso de los juegos para realizar el proceso cognitivo de resolución de problemas (Moreno \& Mayer, 2000; Nilsson \& Jakobsson, 2011), la adopción de juegos en la educación superior (Mozier \& Lean, 2010), los aspectos de implementación de la gamificación y cómo esta se puede utilizar para aprender los métodos científicos, incluida la observación, la explicación, la interpretación y la predicción (Spires et al., 2011; Squire \& Jan, 2007).

- Mejora del proceso educativo: en esta tendencia se adscriben trabajos que se orientan a analizar cómo la gamificación y el aprendizaje activo pueden mejorar la educación (Candido, Murman \& McManus, H. 2007), el uso de elementos de los juegos para hacer los objetivos de aprendizaje más atractivos y alcanzables (Casandra, 2017), y los elementos de los videojuegos que pueden incorporarse en la experiencia de aprendizaje para enriquecerla (Pendry, 2017).

- Cambios en el interés académico en el tema de la gamificación: los principales autores de esta agrupación son Harman, Koohang, Paliszkiewicz y Dickinson (2014).

- Barreras de la adopción de juegos en la educación superior: a esta tendencia se adscribe el trabajo de Moizer, Lean, Towler, y Abbey (2009).

Sin embargo, no existe consenso sobre la definición de gamificación entre los investigadores, ni existe un acuerdo sobre la diferencia entre aprendizaje ABJ y gamificación (Alsawaier, 2018). Comúnmente se señala en la literatura, como principal diferencia entre estos dos conceptos, que la gamificación incorpora los elementos más efectivos de los juegos en el proceso de enseñanza aprendizaje sin comprometerse con un juego específico, mientras el ABJ se basa en un juego para transmitir el contenido al estudiante. A continuación, en la tabla 1 se encontrará de manera más detallada diferentes posturas de autores entorno a las diferencias de estos dos conceptos: 
Tabla 1. Diferencias entre gamificación y aprendizaje basado en juegos

\begin{tabular}{|c|c|c|}
\hline Autores & Gamificación & $\begin{array}{l}\text { Aprendizaje basado en juegos } \\
(A B J)\end{array}$ \\
\hline Keeler (2014) & $\begin{array}{l}\text { Implica el despliegue de elementos del juego } \\
\text { en un entorno fuera de los juegos digitales. }\end{array}$ & $\begin{array}{l}\text { Se realiza cuando los estudiantes jue- } \\
\text { gan juegos para aprender el contenido. }\end{array}$ \\
\hline Issacs (2015) & $\begin{array}{l}\text { Ayuda a crear una atmósfera asociada con el } \\
\text { juego que conduce el aprendizaje. }\end{array}$ & $\begin{array}{l}\text { Se basa en un juego como recipiente } \\
\text { para el aprendizaje de contenido. }\end{array}$ \\
\hline $\begin{array}{l}\text { Simões, Redondo, y } \\
\text { Vilas. (2013) }\end{array}$ & $\begin{array}{l}\text { Utiliza los componentes más efectivos de } \\
\text { los juegos sin comprometerse con un juego } \\
\text { específico, para aumentar los niveles de mo- } \\
\text { tivación y participación en la experiencia de } \\
\text { aprendizaje. }\end{array}$ & $\begin{array}{l}\text { Emplea un juego en específico para el } \\
\text { aprendizaje del contenido. }\end{array}$ \\
\hline Alsawaier (2018) & $\begin{array}{l}\text { Crea un efecto a largo plazo sobre el compro- } \\
\text { miso y la motivación. }\end{array}$ & $\begin{array}{l}\text { El compromiso es de corta duración, } \\
\text { generalmente durante la duración del } \\
\text { juego. }\end{array}$ \\
\hline Alsawaier (2018) & $\begin{array}{l}\text { Funciona al agregar elementos inspirados en } \\
\text { los juegos al entorno del aula, principalmente } \\
\text { para aumentar la motivación, el compromiso y } \\
\text { promover los comportamientos de aprendiza- } \\
\text { je deseados. }\end{array}$ & $\begin{array}{l}\text { Se basa en el uso de juegos para cum- } \\
\text { plir con los resultados de aprendizaje. }\end{array}$ \\
\hline McGonigal (2011) & Defiende la gamificación sobre el ABJ. & $\begin{array}{l}\text { Los juegos educativos son efímeros y } \\
\text { no satisfacen las necesidades de los } \\
\text { estudiantes, de las escuelas y de los } \\
\text { sistemas educativos, en el mejor de los } \\
\text { casos, son una solución temporal. }\end{array}$ \\
\hline $\begin{array}{l}\text { McGonigal (2011) \& } \\
\text { Prensky (2001) }\end{array}$ & $\begin{array}{l}\text { Permite a los estudiantes involucrarse de } \\
\text { principio a fin. }\end{array}$ & $\begin{array}{l}\text { Permite a los estudiantes involucrarse } \\
\text { durante la duración del juego. }\end{array}$ \\
\hline Keven Werbach (2014) & La gamificación es un 'proceso'. & Un juego es un 'producto' \\
\hline
\end{tabular}

Fuente: elaboración propia.

Como se puede evidenciar en la anterior tabla, son muchos los autores que le dan un valor mayor a la gamificación por encima del ABJ, debido a que esta primera permite que el estudiante se involucre de principio a fin en el proceso de aprendizaje, crea un efecto positivo a largo plazo sobre la motivación, el compromiso y los comportamientos de aprendizaje deseados, y no se compromete con un juego específico, sino que toma los principales elementos de estos para conducir el aprendizaje. Teniendo en cuenta las ventajas de la gamificación sobre el ABJ, el presente documento se centrará en el análisis de la gamificación, su concepto, las ventajas de su implementación y los retos que enfrenta un proceso de enseñanza gamificado exitoso. 


\section{Gamificación: concepto, objetivos, aportes y elementos}

La gamificación es un término general utilizado por diferentes personas para referirse a diferentes cosas (Casandra, 2017), "la gamificación podría ser un producto, una forma de pensar, un proceso, una experiencia, una forma de diseño y un sistema, todo a la vez" (Raftopoulos, 2015, citado por Casandra, 2017, p. 158). Así pues, un número representativo de autores la definen como el «uso o utilización» de aspectos propios de los juegos, tales como: mecánicas basadas en juegos, estética y juegos (Kapp, 2012b), mecanismos de juego (Werbach \& Hunter, 2012), mecánica y dinámica del juego (Simões et al.,2013), pensamiento y elementos de juego (Van den Boer, 2013), pensamiento y mecánica del juego (Folmar, 2015), elementos del juego y técnicas de diseño (Kavaliova, Virjee, Maehel \& Kleppe, 2016) y elementos y principios del diseño de juegos (Contreras, 2016).

Mientras, otra corriente de autores consideran que la gamificación es el 'proceso' de a) integrar la teoría y el diseño de los juegos, los elementos del juego, la estética del juego y la mecánica del juego (Ahmed \& Sutton, 2017); b) pensar en el juego y la mecánica del juego para involucrar a los usuarios y resolver problemas (Zichermann \& Cunningham, 2011), c) técnico y artístico que consiste en reproducir objetos, idealizar personajes y entornos (Sánchez, 2015); d) replantear un objetivo de la vida real (Casandra, 2017).

Otros autores se apartan de estas dos corrientes que ven a la gamificación como el 'uso' o como el 'proceso', definiéndola entonces como: un enfoque (Hamari, Koivisto \& Sarsa, 2014), una práctica (Gallego \& De Pablos, 2013), una experiencia (Leaning, 2015), una experiencia inmersiva (Craven, 2015), una práctica empresarial que se encuentra en la intersección entre el marketing, los juegos y la psicología (M2 Research, 2012).

Ahora bien, independientemente de si la gamificación es un uso, un proceso, un enfoque, una práctica, una experiencia, una experiencia inmersiva y/o una práctica empresarial, esta puede tomar lugar en: contextos no relacionados con el juego (Werbach \& Hunter, 2012; Van den Boer, 2013; Leaning, 2015; Contreras, 2016; Simões et al., 2013; Kavaliova et al., 2016), para el ámbito pedagógico en una 'experiencia de aprendizaje' (Ahmed \& Sutton, 2017). De tal modo, tomando los principales aportes de los autores anteriormente mencionados, en el presente documento se entiende por gamificación: el uso, proceso, 
enfoque, práctica y/o experiencia que toma elementos y principios del diseño de juegos en contextos que no son de juego.

Ahora bien, la incorporación de la gamificación en el terreno pedagógico tiene varios objetivos: involucrar a las personas, motivar acciones, promover el aprendizaje y resolver problemas (Kapp, 2012b), acelerar la curva de experiencia, de aprendizaje, la enseñanza de temas complejos y el pensamiento sistémico (Kapp, 2012a), alentar los comportamientos deseados (Werbach \& Hunter, 2012), aumentar el comportamiento objetivo y el compromiso (Van den Boer, 2013), comprender e influir en el comportamiento humano (Dale, 2014), centrarse en las posibilidades de motivación y el cambio en el comportamiento como un resultado (Hamari et al., 2014), crear un entorno visual donde jugar (Sánchez, 2015), cumplir con fines no relacionados con el juego (Folmar, 2015), crear compromiso para el usuario (Craven, 2015), combinar instrucción del área de contenido, alfabetización y habilidades de aprendizaje del siglo 21 en un entorno de aprendizaje altamente atractivo (Kingsley $\&$ Grabner-Hagen, 2015), dar forma a los comportamientos de los usuarios (Kavaliova et al., 2016), replantear un objetivo de la vida real para ser más atractivo y alcanzable (Casandra, 2017), involucrar a los usuarios y resolver problemas (Zichermann \& Cunningham, 2011), crear experiencias de usuario atractivas y emocionantes que involucren al usuario (M2 Research, 2012), mejorar la participación ciudadana, la toma de decisión de los recursos humanos que forman parte de empresas o instituciones y, en definitiva, en la distribución racional y justa del poder (Escribano, 2013), y facilitar una cultura participativa basada en la innovación de agentes internos y externos (Gallego \& De Pablos, 2013). Dentro de los principales aportes de aplicar gamificación en el proceso de enseñanza-aprendizaje se encuentran las siguientes:

1. Promueve la participación de los estudiantes, en tanto que los involucra (Prensky, 2001), y los incentiva a participar en el aula (Deif, 2017).

2. Permite una mejor interiorización y aplicación de los conocimientos adquiridos, pues incentiva la capacidad de resolución de problemas y potencia la internalización de los contenidos de aprendizaje (Harman, Koohang, Paliszkiewicz \& Dickinson, 2014), genera resultados de aprendizaje positivos (Becker, 2007; Gee, 2007), facilita el aprendizaje (Hughes \& Lacy, 2016), aborda muchas de las limitaciones de la enseñanza más tradicional, específicamente problemas de aprendizaje cognitivos y efectivos, y propor- 
ciona a los docentes mejores herramientas para brindar un sentido práctico y aplicado a los estudiantes (Deif, 2017).

3. Crea relajación y motivación (Prensky, 2001), proporciona a los docentes las herramientas necesarias para dirigir y motivar a sus alumnos (Lee \& Hammer, 2011), crea optimismo (Harman, Koohang, Paliszkiewicz \& Dickinson, 2014); tiene un efecto positivo en la motivación de los estudiantes (Kingsley \& Grabner-Hagen, 2015; Leaning, 2015; Papastergiou, 2009; Seaborn \& Fels, 2015; Koivisto \& Hamari, 2014; Alsawaier, 2018).

4. Aumenta el rendimiento de los estudiantes, pues la relajación permite al alumno asimilar las cosas con más facilidad, y la motivación les permite esforzarse sin resentimiento (Prensky, 2001); tiene un efecto positivo en el rendimiento general de los estudiantes (Kingsley y\&- Grabner-Hagen, 2015; Leaning, 2015; Papastergiou, 2009; Seaborn \& Fels, 2015; Koivisto \& Hamari, 2014), mejora el desempeño de los estudiantes (Attali \& Arieli-Attali, 2015).

5. Promueve la interactividad y la colaboración, esto debido a que facilita la interacción social (Harman, Koohang, Paliszkiewicz \& Dickinson, 2014), y facilita la interactividad, la colaboración, el aprendizaje entre pares y el aprendizaje activo (Deif, 2017).

6. Genera y aumenta el compromiso por parte de los estudiantes (Prensky, 2001; Hamari et al., 2014; Kingsley \& Grabner-Hagen, 2015; Leaning, 2015; Papastergiou, 2009; Seaborn \& Fels, 2015; Koivisto \& Hamari, 2014; Seaborn \& Fels, 2015; Alsawaier, 2018), y permite que los estudiantes se entreguen por completo al aprendizaje (Deif, 2017).

7. Ayuda a transformar las actitudes de los estudiantes hacia el aprendizaje (Alsawaier, 2018), aumentando la satisfacción del usuario (Harman, Koohang, Paliszkiewicz \& Dickinson, 2014) y engendrando menos resistencia en el alumno (Hughes \& Lacy, 2016).

8. Es una manera de aprender diferente, en tanto que el aprendizaje se produce de forma natural durante los juegos (Becker, 2007; Gee, 2007), transforma la experiencia de aprendizaje en una experiencia alegre (Lee \& Hammer, 2011), el aprendizaje puede ser divertido (Simões et al., 2013), y es una forma atractiva de aprender (Hughes \& Lacy, 2016). 
La gamificación no puede entenderse de manera integral sin los componentes esenciales de los videojuegos y/o juegos (Alsawaier, 2018). Dentro de estos componentes y/o elementos se encuentran los mencionados a continuación en la tabla 2.

Tabla 2. Principales elementos de los juegos
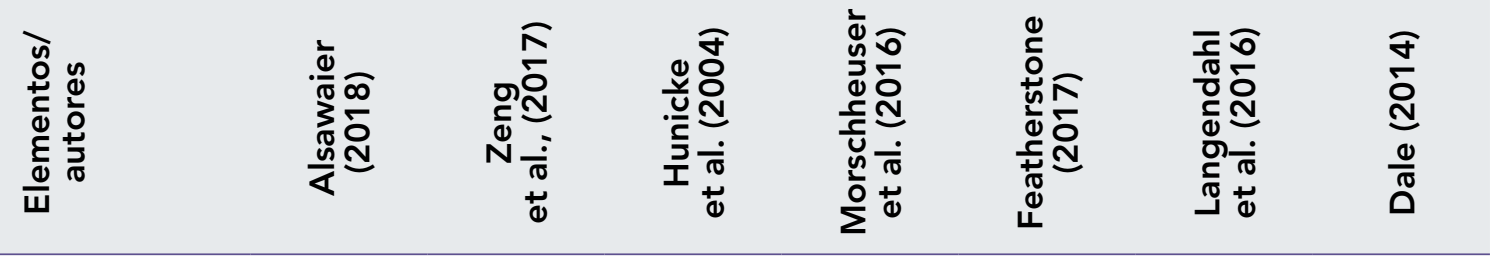

\section{Narrativas, Tematización,} Contexto

$x$

Puntos

Insignias, medallas y trofeos

Niveles

Tabla de clasificación

Misiones y desafíos

Logros

Retroalimentación

Mundo de fantasía/ terri-

torios virtuales

Estado

Competencia

Recompensa

Barra de progreso

Tipo de Jugadores

Avatares

Cuenta regresiva

Fecha límite

Regalo virtual

Autoexpresión

Altruismo

Emoción

Sentido de curiosidad

Resolución de problemas

Autonomía

Dominio

Fuente: elaboración propia.

$\times$

$\times$

$\mathrm{x}$

$x$

X

$x$

X

$\mathrm{X}$

$\mathrm{X}$

$x \quad x \quad x$

$x \quad x \quad x$

$x$

$x$

$x$

X

$x \quad x$

$x$

$x+x$

x $\quad x$

$\mathrm{X}$

X

$x$

$\mathrm{X}$

X

$x$


En función de las características de estos elementos del juego y las similitudes entre ellos Zeng et al. (2017) realizaron una categorización de los elementos del juego en cuatro grupos: recompensa, reconocimiento, progreso y fantasía. Recompensa: se refiere a los elementos del juego que proporcionan recompensas por algunos comportamientos específicos o logros de los participantes, incluidos dinero tangible y regalos, así como bienes virtuales y propiedades del juego. Reconocimiento: se refiere a elementos del juego que soportan un mecanismo donde las acciones de los participantes, para lograr la tarea, se convierten en algún tipo de valores contables con los que comparar, incluyendo puntuación, clasificación, logros y niveles. El progreso: se refiere a los elementos del juego que proporcionan información sobre la progresión de una tarea en curso sobre la premisa de que la realización de un proyecto específico se divide en varias presentaciones o etapas. La fantasía se refiere a los elementos del juego que crean circunstancias virtuales o de fantasía.

Al agrupar los elementos presentados en la tabla 2, atendiendo a las cuatro categorías propuestas por Zeng et al. (2017), se tiene la siguiente conformación (Ver figura 1):

Figura 1. Categorización de los elementos del juego según la propuesta de Zeng et al. (2017)

\begin{tabular}{|c|c|c|c|c|}
\hline Reconocimiento & Fantasía & Progreso & Recompensa & Sin categorizar* \\
\hline $\begin{array}{l}\text { - Puntos } \\
\text { - Insignias, } \\
\text { medallas y } \\
\text { trofeos } \\
\text { - Niveles } \\
\text { - Tabla de } \\
\text { clasificación } \\
\text { - Logros }\end{array}$ & $\begin{array}{l}\text { - Narrativas/ } \\
\text { Tematización/ } \\
\text { Contexto } \\
\text { - Mundo de } \\
\text { fantasía/ } \\
\text { territorios } \\
\text { virtuales } \\
\text { - Avatares }\end{array}$ & $\begin{array}{l}\text { - Misiones y } \\
\text { desafíos } \\
\text { - Estado } \\
\text { - Barra de } \\
\text { progreso }\end{array}$ & $\begin{array}{l}\text { - Recompensa } \\
\text { - Regalo virtual }\end{array}$ & $\begin{array}{l}\text { - Retroalimentación } \\
\text { - Sentido de } \\
\text { curiosidad } \\
\text { - Resolución de } \\
\text { problemas } \\
\text { - Autonomía } \\
\text { - Dominio } \\
\text { - Autoexpresión } \\
\text { - Altruismo } \\
\text { - Emoción } \\
\text { - Cuenta regresiva } \\
\text { - Fecha límite } \\
\text { - Competencia } \\
\text { - Tipo de } \\
\text { jugadores }\end{array}$ \\
\hline
\end{tabular}

*Los elementos denominados 'sin categorizar' no son contemplados en la propuesta de Zeng et al. (2017), por lo cual en los párrafos siguientes se propondrán categorías adicionales a las de este autor para clasificarlos.

Fuente: elaboración propia.

A continuación, se proponen categorías adicionales para agrupar los ítems que no pudieron ser categorizados en ninguno de los cuatro grupos propuestos por Zeng et al. (2017): 
- Personalidad: este ítem informa sobre las características de los jugadores (tipo de jugadores). Este aspecto es clave en un diseño gamificado, pues permite entregar de la manera más adecuada el contenido pedagógico a los estudiantes (Alsawaier, 2018), en tanto que provee información de los jugadores que facilita identificar sus necesidades en un diseño de gamificación pedagógica eficaz (Folmar, 2015), en este ítem se incluye el sentido de curiosidad, en tanto que es una característica que puede identificar a algunos jugadores.

- Duración: dentro de esta categoría se encuentran elementos que señalan la temporalidad de los desafíos, los retos, las misiones, etc., tales como la cuenta regresiva y la fecha límite.

- Interacción social: el altruismo y la competencia hacen parte de esta categoría, ya que de los relacionamientos sociales de los estudiantes pueden surgir comportamientos cooperativos o competitivos dependiendo el tipo de jugador o las reglas de juego.

- Realización personal: los principales componentes de este ítem son la autoexpresión, la emoción, la resolución de problemas, la autonomía, el dominio y la retroalimentación que contribuyen en que el estudiante sienta realización personal al vivenciar el proceso de aprendizaje, en tanto que un proceso de enseñanza gamificado permite que el estudiante se autoexprese de manera natural, libre y espontánea durante el desarrollo del desafío; igualmente, los problemas a resolver en los desafíos, las misiones, los retos, etc. motivan y emocionan al estudiante al permitirle realizar sus propias elecciones y ejercer su autonomía, y la retroalimentación constante del docente guía lo concientiza de su desempeño, lo impulsa a mejorarlo y a dominar los temas abordados.

Vale la pena anotar que, pese a los múltiples aportes y beneficios para el proceso de enseñanza aprendizaje que ofrece la gamificación, en algunos casos ha fracasado su implementación en contextos educativos, esto se ha atribuido principalmente a:

a) La falta de pensamiento de juego cuando se utiliza la gamificación en un contexto educativo (Zichermann, 2010; Folmar, 2015); pues no es suficiente con agregar elementos del juego sin considerar cómo funciona la gamificación, en tanto que el pensamiento del juego exige repensar las prácticas 
de enseñanza. Así pues, en los métodos de enseñanza tradicionales, los estudiantes obtienen sus calificaciones en función de la ejecución de una tarea, ya que demuestran el logro, mientras que en la gamificación no se recompensa el ganador sino el esfuerzo, con insignias o puntos incluso cuando el objetivo no se completa (Folmar, 2015). En otras palabras, en un ambiente gamificado, se alienta a los estudiantes a involucrarse en el proceso y se los evalúa en consecuencia, independientemente de si tuvieron éxito en su tarea o no. Por supuesto, esto no es para socavar la importancia de la tarea sino más bien para motivar a los estudiantes a esforzarse en abordar los diferentes desafíos de aprendizaje (Alsawaier, 2018).

b) La base de conocimiento y la investigación empírica que conecta la gamificación con los principios teóricos es escasa (Alsawaier, 2018); esto es corroborado por Seaborn y Fels (2015), quienes hicieron una revisión de la literatura sobre gamificación, en la cual analizaron treinta y dos (32) estudios, de los cuales solo diez (10) de ellos fueron fundados en teorías (cinco de los cuales pertenecen a un mismo autor) y el resto no tenía conexión con fundamentos teóricos. Además, existe una brecha entre la teoría y la práctica, donde la teoría no se examina empíricamente en el contexto de gamificación y el trabajo aplicado carece de referencia a la teoría (Seaborn \& Fels, 2015).

Ante este escenario, resulta clave preguntarse ¿cómo implementar de manera exitosa la gamificación?, lo cual se abordará a continuación.

\section{2. ¿Cómo implementar exitosamente la gamificación en el contexto educativo?}

Para evitar el fracaso en la implementación de la gamificación en contextos educativos, un diseño gamificado debe contemplar los siguientes aspectos:

1. Promover la motivación: los diseñadores de juego y/o educadores deben preparar misiones para aumentar la motivación de los participantes (Kolb, 
2015), para ello, los diseñadores deben analizar a los jugadores y preguntarse sobre qué los motiva (Raftopoulos, 2017; citado en Pappas, 2017), es decir, es importante entender cómo y por qué se logra la motivación (Gee, 2005). La pregunta es cómo utilizar los elementos del juego de una manera que sea motivadora, en lugar de desmotivadora para una amplia gama de alumnos, en tanto que aburrirse o sentirse rezagados (incompetentes y abrumados) en la escuela son las dos razones más importantes para abandonar (Azzam, 2007). A diferencia de otros factores socioeconómicos y personales que afectan las tasas de deserción, estas son dos áreas donde los educadores sí tienen influencia. Cualquier etiqueta que coloquemos en estos productos como juegos, o no, lo que tienen en lo común es un enfoque justificable en el compromiso emocional del jugador / alumno (Azzam, 2007).

2. Permitir la elección y la autonomía: el diseño gamificado debe permitir la modificación, es decir, permitir a los usuarios hacer sus propias elecciones de juego, facultarlos para elegir sus propios avatares, crear misiones y decidir involucrar el contenido gamificado individualmente o trabajando en equipo (Kolb, 2015). Es clave que se permita la elección y la autonomía del aprendizaje autodirigido en la experiencia gamificada, esto debido a que la autonomía, como uno de los tres principios de la teoría de autodeterminación, está conectada a la motivación intrínseca. De modo que, al permitir que el alumno tome decisiones en la experiencia gamificada, se posibilitará que se produzca una gamificación significativa (Gibson \& Jakl, 2015; Nicholson, 2015). De esta manera, es indispensable empoderar a los estudiantes (Gee, 2005). Ahora bien, los videojuegos permiten recompensas abiertas y dirigidas por los estudiantes, y un profesor que se encuentre realizando un diseño educativo gamificado podría lograr lo mismo otorgando a un alumno una recompensa ad hoc, por las actividades ad hoc que los estudiantes realicen a su propia discreción (Shpakova, Dörfler \& Macbryde, 2016). Otro punto a considerar es que a los jugadores les gusta tener autonomía para elegir su propio nivel de experiencia y que puedan trabajar a su propio ritmo (Gee, 2005), de tal modo la autonomía es un elemento del juego que contribuye a la gamificación efectiva (Dale, 2014).

3. Promover la colaboración, la interacción social y la competencia: la creación de un diseño de juego interactivo y exitoso debe conducir a la colaboración 
(Lawley, 2012; Zhang \& Clear, 2015) y el trabajo en equipo mediante el uso de tablas de clasificación o gremios (Gee, 2005). Los diseños gamificados exitosos que incorporan la colaboración ayudan a la aparición de comportamientos positivos de aprendizaje (Zhang \& Clear, 2015), a medida que los jugadores colaboran, se involucran en una actividad compartida, relevante y orientada a objetivos (Kim, Glassman \& Williams, 2015). La mecánica del juego que promueve la interacción social y la competencia es la mejor manera de lograr los objetivos de aprendizaje de un proyecto (Hughes, 2017; citado en Pappas, 2017), un ejemplo de cómo incorporar la competencia de manera positiva es permitir a los estudiantes compartir sus experiencias en foros de intercambio como Facebook o YouTube, discusiones en clase o publicaciones en blogs (Featherstone, 2017).

4. Redefinir el fracaso y permitir 'prueba y error': el diseño exitoso del juego permite a los jugadores probar varias veces para lograr el éxito, de modo que en un entorno gamificado el fracaso se redefine, dejando de ser visto como un retroceso, convirtiéndose más bien en una oportunidad para aprender de los errores y corregirlos (Hanus \& Fox, 2015), es decir, en un camino hacia el éxito (Gee, 2014). Este cambio en la concepción del fracaso permite incorporar la competencia de manera positiva al permitirse el crear un juego que nunca termina, permitiendo oportunidades para muchos ganadores (Featherstone, 2017). Así mismo, los estudiantes que realizan actividades ad hoc a su propia discreción ganan por hacer las cosas bien en lugar de perder por cometer errores, y sienten que reciben un tratamiento más equitativo (Shpakova et al., 2016). En este sentido, la libertad para fallar ejemplifica una forma de evaluación formativa, y una vez dominado el desafío, aparecen nuevos problemas para resolver, nuevos desafíos (Langendahl, Cook \& Mark-Herbert, 2016; Myers \& Reigeluth, 2016).

5. Llevar a que el jugador reflexione y experimente las consecuencias de las elecciones: la gamificación en el aprendizaje funciona mejor cuando se hace que el alumno piense y experimente las consecuencias de sus elecciones (Coppens, 2017; citado en Pappas, 2017). La exploración y la exposición a las consecuencias de las decisiones fomenta el aprendizaje (Langendahl 
et al., 2016), y en caso de que fallen, los estudiantes reconsideran y aprenden de nuevo hasta adquirir el dominio (Langendahl et al., 2016; Myers \& Reigeluth, 2016).

6. Retroalimentar al jugador: los jugadores que reciben comentarios constructivos después del fracaso en el entorno gamificado expresan emociones positivas sobre su experiencia (Herzig et al., 2015), igualmente la retroalimentación mejora el compromiso y la motivación de los estudiantes (Langendahl et al., 2016), y cuando la retroalimentación es inmediata contribuye a una gamificación efectiva (Dale, 2014).

7. Agregar elementos atractivos del diseño de juegos a la instrucción: esto ayuda significativamente a captar la atención de aquellos que tienen dificultades para concentrarse en el aprendizaje en el entorno normal y crea una asociación positiva con aprendizaje (Meredith, 2017; citado en Pappas, 2017).

8. Establecer parámetros de competencia equilibrados: la competencia puede ser perjudicial para los alumnos con bajo rendimiento y baja autoeficacia. De ahí que los videojuegos abordan el síndrome de "no ganar me hace sentir mal" al evaluar automáticamente a los jugadores y al nivelarlos para que los jugadores con igual equilibrio compitan y se sientan menos desmotivados cuando pierden (Andrade et al., 2016). Así pues, existen algunas formas de incorporar la competencia de manera positiva: a) crear un juego que nunca termina, permitiendo oportunidades para muchos ganadores; b) emparejar jugadores con niveles de habilidad similares para que el juego se dispute de cerca, se gane duro y sea satisfactorio para todos; c) permitir que los estudiantes compitan consigo mismos al apostar puntos adicionales a su calificación en base a las respuestas a las preguntas de la clase; y d) permitir a los estudiantes compartir sus experiencias en foros de intercambio como Facebook o YouTube, discusiones en clase o publicaciones en blogs (Featherstone, 2017).

9. Proporcionar un espacio seguro para el andamiaje educativo: los videojuegos proporcionan lo que Gee (2005) denominó ‘tanques de peces'. La teoría detrás de las peceras es que los aprendices aprenden acerca de un sistema simplificado antes de que el juego los exponga a un sistema más complejo, como una pecera antes que el ecosistema del océano. Esta estrategia permite 
al alumno familiarizarse con los conceptos básicos y observar patrones que le permitirán reconocer aquello a lo que debe prestar atención. Las sugerencias para usar esta estrategia fuera de los videojuegos incluyen el desarrollo de problemas bien ordenados que usan modelos proporcionados, teorías y herramientas inteligentes (Gee, 2005). Otro elemento esencial para la resolución de problemas que ofrecen los videojuegos es un lugar seguro para que los alumnos experimenten la autenticidad y el logro, a esto Gee (2005) lo denominó “caja de arena”, lo que significa que la instrucción permite que el fracaso sea informativo y no punitivo y se fomente la creatividad y la asunción de riesgos para mejorar la comprensión. Por su parte, Myers y Reigeluth (2016) elogiaron la capacidad de los videojuegos para proporcionar técnicas seguras de andamiaje antes de que los estudiantes sean colocados en situaciones críticas en el mundo real.

10. Promover el compromiso: los diseñadores de juego y/o educadores deben preparar misiones para aumentar el compromiso (Kolb, 2015).

11. Permitir la progresión y el dominio por parte del jugador: la progresión mejora el compromiso y la motivación de los estudiantes (Langendahl et al., 2016), y el dominio es un elemento del juego que contribuye a la gamificación efectiva (Dale, 2014).

12. Alinear con los principios pedagógicos y contribuir en el logro de los objetivos de aprendizaje: el énfasis no debe estar de ninguna manera en adicionar elementos del juego a expensas del aprendizaje de estudiantes, en tanto que un diseño gamificado defectuoso o superficial puede llevar a que se pierda el interés y el compromiso por parte de los estudiantes (Lawley, 2012), de modo que los objetivos de aprendizaje deben estar bien planteados y presentados explícitamente antes de incluir elementos de gamificación, y estos a su vez deben ser integrados en el diseño gamificado (Kolb, 2015).

13. Plantear problemas a resolver por los jugadores: la resolución de problemas es un elemento del juego que contribuye a la gamificación efectiva (Dale, 2014), por lo cual, la identificación clara del problema a solucionar asegurará que los estudiantes entiendan las habilidades necesarias para lograr sus objetivos y luego puedan poner en práctica esas habilidades como un conjunto (Gee, 2005). 
14. Despertar el sentido de curiosidad: Dale (2014) señala que el sentido de curiosidad es un elemento del juego que contribuye a una gamificación efectiva.

15. Usar plataformas o herramientas de apoyo: Kolb (2015) recomienda incorporar herramientas prácticas en el diseño gamificado del entorno de aprendizaje, como el uso de un software de gamificación confiable que permita el despliegue completo de las funciones de gamificación (por ejemplo: 3DGamelab, Gradecraft y Classcraft), en cualquier diseño gamificado con contenido de aprendizaje.

Ahora bien, teniendo en cuenta que la carencia de estudios en donde se vincule la aplicación de gamificación educativa con los fundamentos teóricos y/o principios de la gamificación, y la proliferación de trabajos aplicados carentes de referencia a bases teóricas que validen la aplicación de la gamificación realizada, son aspectos que han llevado al fracaso de la gamificación en contextos educativos, como se había mencionado en párrafos anteriores, en el presente documento se busca contribuir a solventar estas falencias conectando la aplicación de la gamificación educativa realizada en el programa de Contaduría Pública a Distancia de la Universidad Militar Nueva Granada con los fundamentos teóricos identificados en los apartados anteriores, buscando con ello aportar en la reducción de la brecha existente entre teoría y práctica, permitiendo que la teoría se contraste de manera empírica en un caso aplicado.

De tal modo, se tomaron los elementos de los juegos y los aspectos claves a tener en cuenta para una implementación exitosa de la gamificación en el contexto educativo (ambos ítems abordados en los apartados anteriores) para realizar la aplicación de gamificación educativa durante el primer y segundo semestre de 2017 en los cursos Contabilidad II (segundo semestre), presupuestos y finanzas públicas (sexto semestre), Administración financiera (sexto semestre) y Contabilidad pública (séptimo semestre). Los resultados de esta experiencia se relacionan a continuación. 


\section{Aplicación de Gamificación en el programa de Contaduría Pública a Distancia de la Universidad Militar Nueva Granada}

En el proceso de implementación de la gamificación se hizo uso de las aulas virtuales en Moodle de la Universidad Militar Nueva Granada (UMNG) y se incorporaron los elementos propios de los juegos que se presentan en la tabla 3:

Tabla 3. Elementos del juego incorporados en la experiencia de gamificación educativa

\begin{tabular}{llcccc}
\hline No. & Elementos de los juegos & $\begin{array}{c}\text { Incorporación en } \\
\text { la experiencia }\end{array}$ & No. & $\begin{array}{c}\text { Elementos de los } \\
\text { juegos }\end{array}$ & $\begin{array}{c}\text { Incorporación } \\
\text { en la } \\
\text { experiencia }\end{array}$ \\
\hline 1 & Narrativas/ Tematización/contexto & $\checkmark$ & 14 & Tipo de Jugadores & X \\
\hline 2 & Puntos & $\checkmark$ & 15 & Avatares & $\checkmark$ \\
\hline 3 & Insignias, medallas y trofeos & $\checkmark$ & 16 & Cuenta regresiva & $\checkmark$ \\
\hline 4 & Niveles & $\checkmark$ & 17 & Fecha límite & $\checkmark$ \\
\hline 5 & Tabla de clasificación & $\checkmark$ & 18 & Regalo virtual & $\checkmark$ \\
\hline 6 & Misiones y desafíos & $\checkmark$ & 19 & Autoexpresión & $\checkmark$ \\
\hline 7 & Logros & $\checkmark$ & 20 & Altruismo & $\checkmark$ \\
\hline 8 & Retroalimentación & $\checkmark$ & 21 & Emoción & $\checkmark$ \\
\hline 9 & Mundo de fantasía/ territorios virtuales & $X$ & 22 & Sentido de curiosidad & $\checkmark$ \\
\hline 10 & Estado & $X$ & 23 & Resolución de pro- & $\checkmark$ \\
\hline 11 & Competencia & $\checkmark$ & 24 & Autonomía & $\checkmark$ \\
\hline 12 & Recompensa & $\checkmark$ & 25 & Dominio & $\checkmark$ \\
\hline 13 & Barra de progreso & $\times$ & & $\checkmark$ \\
\hline
\end{tabular}

$\checkmark$ : Elementos que fueron incorporados $X$ : Elementos que no fueron incorporados

Fuente: elaboración propia.

1) Narrativas/Tematización/Contexto y 23) Resolución de problemas: Se creó un storytelling en las asignaturas mencionadas con los ítems que se relacionan en la tabla 4. El storytelling aplicado en la presente investigación consistió en contar historias cuyo escenario es el ámbito empresarial, para ello se realizaron historietas en Pixton que transmitieran información y conocimientos a los estudiantes de las temáticas abordadas en las asignaturas y al finalizar cada historia se les proponía unos retos para colaborar en resolución de un problema planteado en la misión, involucrando de esta manera a estudiantes y docentes como personajes dentro del storytelling. 
Tabla 4. Storytelling creados

\begin{tabular}{|c|c|c|}
\hline Historia creada & $\begin{array}{c}\text { Presupuestos } \\
\text { y finanzas Públicas }\end{array}$ & Administración financiera \\
\hline $\begin{array}{l}\text { Situación } \\
\text { problemática }\end{array}$ & $\begin{array}{l}\text { A nivel mundial existe una crisis } \\
\text { en los presupuestos y finanzas } \\
\text { públicas }\end{array}$ & $\begin{array}{l}\text { La empresa Eureka Ltda. informa a los miembros del comité } \\
\text { financiero que las organizaciones con las que tiene vínculos } \\
\text { comerciales se encuentran inmersas en graves escándalos } \\
\text { financieros, lo cual puede afectar directamente las finanzas y } \\
\text { reputación de la empresa }\end{array}$ \\
\hline $\begin{array}{l}\text { Rol asignado a } \\
\text { los estudiantes }\end{array}$ & $\begin{array}{l}\text { Ciberhéroes con poderes para } \\
\text { salvar al mundo de la crisis } \\
\text { financiera y presupuestaria en } \\
\text { la cual se encuentra, debido a } \\
\text { sus conocimientos contables y } \\
\text { financieros. }\end{array}$ & $\begin{array}{ll}\text { Miembros del comité financie- } & \text { Miembros del comité financie- } \\
\text { ro (gerentes) de Eureka Ltda., } & \text { ro (gerentes) de Eureka Ltda., } \\
\text { encargados de evaluar las } & \text { encargados de evaluar la } \\
\text { finanzas de la organización y } & \text { situación financiera actual de } \\
\text { enfrentar los riesgos financie- } & \text { la organización. } \\
\text { ros que se avecinan } & \end{array}$ \\
\hline Rol docente & $\begin{array}{l}\text { Investigadora y encargada de } \\
\text { liderar al equipo de ciberhé- } \\
\text { roes en el cumplimiento de la } \\
\text { misión }\end{array}$ & Presidenta de la empresa Eureka Ltda. \\
\hline
\end{tabular}

Fuente: elaboración propia.

2) Puntos, 3) Insignias, medallas y trofeos, 7) Logros, 12) Recompensa, y 18) Regalo virtual: en lo correspondiente al establecimiento de recompensas mediante la asignación de medallas, trofeos y puntos, se utilizaron rúbricas de evaluación en las cuales se les aclaró a los estudiantes que el trabajo realizado en el "acondicionamiento" y en el "reto" sería valorado mediante la asignación de medallas para reconocer el importante trabajo realizado. Se estableció la medalla de oro (50 puntos) para aquellos que cumplieran con excelencia los criterios de calificación, la medalla de plata (40 puntos) para quienes tengan un desempeño sobresaliente a la luz de los criterios de calificación, 1 up- vida extra (20 puntos) para aquellos que tengan un desempeño insuficiente a la luz de los criterios de calificación, y un llamado de atención ( 0 puntos) para aquellos que por algún motivo no realicen el acondicionamiento ni enfrenten el reto. También se estableció un sistema de recompensas por esfuerzo meritorio en el cual se tenía en cuenta el puntaje total en todas las misiones y si se evidenciaba un desempeño excelente de algún o algunos grupos se le otorgaba un trofeo de oro o plata, lo cual implicaría una ganancia de puntos extra sobre las calificaciones obtenidas. 
4)Niveles y 6) Misiones y desafíos: en lo respectivo a los retos, misiones y/o desafíos en cada una de las asignaturas se diseñaron tres misiones, una por cada corte ${ }^{1}$ y una misión final acumulativa. Cada misión (exceptuando la final) se encuentra compuesta de: un acondicionamiento de preparación y un reto que se evalúan de manera separada.

En cada "acondicionamiento" se buscó que la actividad diseñada propendiera porque el estudiante comprendiera e interiorizara conceptos e ideas claves de la temática abordada, en estas actividades el estudiante debía realizar infografías y videos, con el fin de plasmar sus conocimientos de manera dinámica respecto al tema abordado en las lecturas asignadas en cada acondicionamiento. Mientras en cada "reto" se buscó que los estudiantes pusieran en práctica los conocimientos adquiridos mediante la realización de ejercicios matemáticos orientados a la toma de decisiones, o ejercicios de argumentación en donde el estudiante debía realizar análisis crítico de la información del acondicionamiento y confrontarla con artículos académicos de revistas indexadas con el fin de resolver el reto asignado, estos cálculos, análisis y conclusiones debían ser presentados en una exposición oral grabada y enviada en un prezi con voz.

20) Altruismo: de otro lado, en lo respectivo al trabajo colaborativo al interior de los grupos para la realización de los acondicionamientos de preparación y el cumplimiento de los retos, se conformaron grupos de tres personas. La asignación de los grupos fue realizada de manera automática haciendo uso de la plataforma de Moodle.

5) Tabla de clasificación y 11) Competencia: en lo relativo a la competencia grupal, con base en los criterios de evaluación de la rúbrica de calificación elaborada para cada reto y acondicionamiento, se procedió a revisar el puntaje total de cada grupo en cada una de las misiones y ordenar los puntajes del mayor al menor y publicarlos en el aula virtual en un archivo llamado "tabla de posiciones", en el cual los estudiantes podían visualizar el logo del grupo, la posición frente al resto de equipos, la medalla y el puntaje obtenidos en cada misión. Igualmente, después de publicar la tabla de posiciones también se publicaba mediante la herramienta de Pixton una felicitación pública, en la parte superior de Moodle, al grupo que obtuvo el mejor desempeño en la misión, esto con el fin de exaltar

1 En el programa de Contaduría Pública a Distancia de la uMNG una materia se estructura en 3 cortes de evaluación para completar una nota definitiva al final del semestre. 
el desempeño de este grupo e incentivar al resto de equipos a querer aparecer en los primeros lugares de la tabla de posiciones y recibir en el futuro una felicitación similar.

8) Retroalimentación: después de cada acondicionamiento y reto los estudiantes recibían retroalimentación inmediata de los resultados obtenidos por el aula virtual en Moodle, igualmente en las videoconferencias semanales también se retroalimentaban los resultados obtenidos y los aspectos por mejorar.

15) Avantares, 19) Autoexpresión y 24) Autonomía: la creación del logo del grupo y la creación de los personajes por parte de los estudiantes fueron evaluados dentro del ítem de creatividad en todas las entregas, la creación de estos se realizó de manera libre y espontanea por parte de los estudiantes, de modo que podían desplegar toda su autonomía, imaginación e innovación, en tanto que no había normas para la creación del logo y de los personajes.

17) Fecha límite, 19) Autoexpresión y 24) Autonomía: todos los acondicionamientos y retos tenían una fecha máxima de entrega, en la cual debían ser enviados por el aula virtual de Moodle. Estas fechas se informaban desde la primera clase para que los estudiantes tuvieran el tiempo suficiente para realizarlas en sus respectivos equipos y haciendo uso de su autonomía podían entregarlas en cualquier momento antes de esa fecha. Igualmente, la autoexpresión y la autonomía se vivencian en cada una de las misiones en donde hay unos retos que resolver y los estudiantes seleccionan la mejor manera de hacerlo.

15) Usar plataformas o herramientas de apoyo: teniendo en cuenta que la educación a distancia requiere de estudio independiente, trabajo colaborativo y horas de acompañamiento tutorial. En las videoconferencias semanales realizadas a cada grupo por la plataforma Blackboard Collaborate se implementó la herramienta Kahoot. Esta herramienta también fue utilizada para el establecimiento de un procedimiento de competición individual que requería que los estudiantes, de manera independiente, trabajaran las lecturas con antelación para poder responder adecuadamente las preguntas planteadas en el kahoot, de tal modo la docente compartía entre 12 y 16 preguntas en esta herramienta, los estudiantes a nivel individual debían resolver dichas preguntas en tiempo real. Cabe mencionar que en Kahoot es posible visualizar después de cada pregunta los resultados de los estudiantes con los mejores puntajes, con base en el acierto a la pregunta y menor 
tiempo en responder, por lo cual después de resolver cada pregunta los estudiantes podían visualizar si habían fallado o acertado en la pregunta y la posición en que se ubicaban respecto a los demás participantes. Otro punto importante, es que después de mostrar la respuesta correcta a cada pregunta, la docente procedía a explicar las razones por las cuales la respuesta era la correcta y las demás respuestas eran erróneas, del mismo modo se aprovechaba para preguntar a los estudiantes por qué seleccionaron determinada opción buscando que los estudiantes argumentaran su selección y mostrarán ejemplos o casos para complementar las respuestas, de modo que tanto la docente como los estudiantes tuvieran un rol activo en la sesión de videoconferencia.

La materialización de los ítems: 21) Emoción, 22) Sentido de curiosidad y 25) Dominio, se puede visualizar en la tabla 5.

Para analizar la percepción de los estudiantes respecto a la efectividad de la aplicación de la "gamificación" se elaboró una encuesta mediante un formulario en Google Drive, la cual debía ser contestada por los estudiantes al finalizar el semestre como parte de las actividades calificables del último corte de notas.

En cuanto al porcentaje de participación estudiantil frente al número de estudiantes inscritos en las asignaturas en donde se aplicó la "gamificación", se tiene que de 117 estudiantes inscritos, 104 contestaron las encuestas, obteniendo así un porcentaje de respuesta del 89\%. A continuación, se procederá a realizar el análisis de la información recolectada.

Respecto a la primera pregunta sobre cómo calificaron la aplicación de "gamificación" en la asignatura (excelente, muy bueno, bueno, regular, malo) y la segunda pregunta sobre la opinión de los estudiantes sobre si la Gamificación facilita el aprendizaje e interiorización de contenidos, se tiene que en ambos casos (pregunta 1 y pregunta 2) el 94\% de los estudiantes valoraron como buena y excelente la aplicación y utilidad de gamificación educativa.

Para entender con mayor profundidad estos resultados, se agruparon las principales razones que dieron los estudiantes para valorar positivamente la aplicación de la gamificación educativa (tabla 5), y estas a su vez fueron contrastadas con los elementos del juego incorporados en la experiencia de gamificación educativa mencionados en el apartado 


\section{1 y los aspectos clave para realizar una implementación exitosa de la gamificación en el contexto educativo abordados en el apartado 2.}

Tabla 5. Principales razones dadas por los estudiantes para valorar positivamente la aplicación de gamificación educativa realizada en 2017

\begin{tabular}{|c|c|c|}
\hline Razones dadas los estudiantes & $\begin{array}{c}\text { Aspecto clave para realizar una implementación } \\
\text { exitosa de la gamificación en el contexto } \\
\text { educativo, con el que se relaciona }\end{array}$ & $\begin{array}{l}\text { Elemento del } \\
\text { juego, con el } \\
\text { que se relaciona }\end{array}$ \\
\hline $\begin{array}{l}\text { Muestra con un lenguaje claro las } \\
\text { actividades y procedimientos enca- } \\
\text { minados hacia una labor docente } \\
\text { eficiente y eficaz }\end{array}$ & 13. Plantear problemas a resolver por los jugadores & $\begin{array}{l}\text { 1) Narrativas, } \\
\text { Tematización, } \\
\text { Contexto } \\
\text { 23) Resolución de } \\
\text { problemas }\end{array}$ \\
\hline $\begin{array}{l}\text { Invita a los involucrados en el pro- } \\
\text { ceso de enseñanza-aprendizaje a } \\
\text { divertirse y despertar su creatividad }\end{array}$ & $\begin{array}{l}\text { 1. Promover la motivación } \\
\text { 7. Agregar elementos atractivos del diseño de juegos a } \\
\text { la instrucción }\end{array}$ & 21. Emoción \\
\hline $\begin{array}{l}\text { Es una forma diferente de aprender, } \\
\text { comprender y apropiarse de los } \\
\text { conceptos }\end{array}$ & 14. Despertar el sentido de curiosidad & $\begin{array}{l}\text { 22. Sentido de } \\
\text { curiosidad }\end{array}$ \\
\hline $\begin{array}{l}\text { Las actividades se vuelven más } \\
\text { divertidas con el fin de mejorar en } \\
\text { cada una, se dan premios a los me- } \\
\text { jores y esto hace que el estudiante } \\
\text { se esfuerce por presentar un buen } \\
\text { contenido aprendiendo }\end{array}$ & $\begin{array}{l}\text { 1. Promover la motivación } \\
\text { 7. Agregar elementos atractivos del diseño de juegos a } \\
\text { la instrucción } \\
\text { 10. Promover el compromiso }\end{array}$ & $\begin{array}{l}\text { 3. Insignias, meda- } \\
\text { Ilas y trofeos } \\
\text { 7. Logros } \\
\text { 12. Recompensa } \\
\text { 18. Regalo virtual } \\
\text { 21. Emoción }\end{array}$ \\
\hline $\begin{array}{l}\text { El hecho de querer estar en la tabla } \\
\text { de posiciones de la asignatura en un } \\
\text { primer lugar hace exigir más a nivel } \\
\text { personal }\end{array}$ & $\begin{array}{l}\text { 1. Promover la motivación } \\
\text { 3. Promover la colaboración, la interacción social y la } \\
\text { competencia } \\
\text { 7. Agregar elementos atractivos del diseño de juegos a } \\
\text { la instrucción } \\
\text { 10. Promover el compromiso }\end{array}$ & $\begin{array}{l}\text { 5.Tabla de clasifi- } \\
\text { cación } \\
\text { 7.Logros } \\
\text { 11.Competencia } \\
\text { 12. Recompensa } \\
\text { 21.Emoción }\end{array}$ \\
\hline $\begin{array}{l}\text { Los trabajos o actividades pro- } \\
\text { puestos resultan interactivos y de } \\
\text { profundización en la investigación } \\
\text { para sustentarlos }\end{array}$ & $\begin{array}{l}\text { 1. Promover la motivación } \\
\text { 5. Llevar a que el jugador reflexione y experimente las } \\
\text { consecuencias de las elecciones } \\
\text { 7. Agregar elementos atractivos del diseño de juegos a } \\
\text { la instrucción } \\
\text { 11. Permitir la progresión y dominio por parte del jugador }\end{array}$ & $\begin{array}{l}\text { 6.Misiones y de- } \\
\text { safíos } \\
\text { 21.Emoción } \\
\text { 23. Resolución de } \\
\text { problemas }\end{array}$ \\
\hline $\begin{array}{l}\text { Mejora la productividad, ayuda a la } \\
\text { participación y la colaboración }\end{array}$ & $\begin{array}{l}\text { 3. Promover la colaboración, la interacción social y la } \\
\text { competencia } \\
\text { 10. Promover el compromiso } \\
\text { 11. Permitir la progresión y dominio por parte del jugador }\end{array}$ & $\begin{array}{l}\text { 20.Altruismo } \\
\text { 25.Dominio }\end{array}$ \\
\hline
\end{tabular}




\section{Razones dadas los estudiantes Aspecto clave para realizar una implementación exitosa de la gamificación en el contexto educativo, con el que se relaciona}

Hace que el material se recuerde de forma divertida y gráfica, uniendo los temas y generalizándolos como un todo

1. Promover la motivación

7. Agregar elementos atractivos del diseño de juegos a la instrucción

12. Alinear con los principios pedagógicos y contribuir en el logro de los objetivos de aprendizaje

13. Plantear problemas a resolver por los jugadores
Exige averiguar y leer para entender los temas

\section{Permitir la elección y la autonomía}

10. Promover el compromiso

12. Alinear con los principios pedagógicos y contribuir en el logro de los objetivos de aprendizaje

13. Plantear problemas a resolver por los jugadores

Cada estudiante se responsabiliza de investigar los temas de la materia

\section{Permitir la elección y la autonomía}

10. Promover el compromiso
12. Alinear con los principios pedagógicos y contribuir en el logro de los objetivos de aprendizaje 13. Plantear problemas a resolver por los jugadores

Por medio de juegos didácticos nuestro cerebro absorbe más fácil los conocimientos

Se brindan ejemplos de situaciones reales y facilita entender y aplicar los temas estudiados
7. Agregar elementos atractivos del diseño de juegos a la instrucción
6. Retroalimentar al jugador

9. Proporcionar un espacio seguro para el andamiaje educativo
Elemento del juego, con el que se relaciona

1.Narrativas/

Tematización/

Contexto

21.Emoción

25.Dominio

23. Resolución de problemas

24.Autonomía

25. Dominio

23. Resolución de

problemas

24.Autonomía

25.Dominio

\section{Emoción}

1.Narrativas/

Tematización/

Contexto

11. Permitir la progresión y dominio por parte del jugador

12. Alinear con los principios pedagógicos y contribuir

23. Resolución de

en el logro de los objetivos de aprendizaje

problemas

25.Dominio

En algunos momentos la teoría

1. Promover la motivación

21.Emoción

cansa, pero lo didáctico llama la

7. Agregar elementos atractivos del diseño de juegos a

la instrucción

22. Sentido de

14. Despertar el sentido de curiosidad

curiosidad

\begin{tabular}{l} 
Al ser algo diferente se vuelve \\
interesante \\
\hline Utilizo en mi vida diaria, laboral, \\
personal o estudiantil parte de mi \\
memoria fotográfica, e implementar \\
actividades de este tipo facilita \\
recordar mejor
\end{tabular}

14. Despertar el sentido de curiosidad

22. Sentido de curiosidad

7. Agregar elementos atractivos del diseño de juegos a 25.Dominio la instrucción

11. Permitir la progresión y dominio por parte del jugador

Exige retroalimentarse de todo el contenido y resumirlo para exposición a la docente permitiendo aprender muy bien las temáticas
2. Permitir la elección y la autonomía

10. Promover el compromiso

11. Permitir la progresión y dominio por parte del jugador

12. Alinear con los principios pedagógicos y contribuir en

el logro de los objetivos de aprendizaje

13. Plantear problemas a resolver por los jugadores
23. Resolución de problemas

24.Autonomía

25.Dominio

Fuente: elaboración propia. 
Como se puede visualizar en la tabla 5, en la implementación de gamificación educativa realizada se logró incorporar varios de los quince aspectos identificados en el presente trabajo como claves para una implementación exitosa de la gamificación, quedando por abordar los aspectos relacionados con: Redefinir el fracaso y permitir 'prueba y error' y establecer parámetros de competencia equilibrados, por tanto se recomienda para futuras investigaciones el estudio de la implementación de estos dos aspectos en un proceso gamificado para evaluar su efectividad y aporte.

De otro lado, se incorporaron varios de los elementos de juegos identificados y categorizados en el presente estudio, quedando por adicionar solamente: mundo de fantasía/ territorios virtuales, estado, barra de progreso, tipo de jugadores, y cuenta regresiva, por tanto, también se exhorta a su abordaje en futuros trabajos en donde se busque solventar la brecha existente entre la teoría y la práctica.

A partir de las razones que dan los estudiantes para valorar positivamente la gamificación educativa implementada, se validan los aportes de la gamificación identificados en la teoría, estos son: promueve la participación de los estudiantes, permite una mejor interiorización y aplicación de los conocimientos adquiridos, crea relajación y motivación, aumenta el rendimiento de los estudiantes, promueve la interactividad y la colaboración, esto debido a que facilita la interacción, genera y aumenta el compromiso por parte de los estudiantes, ayuda a transformar las actitudes de los estudiantes hacia el aprendizaje, es una manera de aprender diferente que transforma la experiencia de aprendizaje en una experiencia alegre, atractiva y divertida

\section{Conclusiones}

Los principales elementos de juego que se deben tener en cuenta cuando se realiza un diseño gamificado en contextos educativos son: narrativas/ tematización/contexto, puntos, insignias, medallas y trofeos, niveles, tabla de clasificación, misiones y desafíos, logros, retroalimentación, mundo de fantasía/ territorios virtuales, estado, competencia, recompensa, barra de progreso, tipo de jugadores, avatares, cuenta regresiva, fecha límite, regalo virtual, autoexpresión, altruismo, emoción, sentido de curiosidad, resolución 
de problemas, autonomía y dominio. Estos elementos se pueden agrupar en las siguientes grandes categorías: recompensa, reconocimiento, progreso, fantasía, personalidad, duración, interacción social y realización personal.

La falta de pensamiento de juego cuando se utiliza la gamificación en un contexto educativo y la escasez de trabajos de gamificación aplicados en donde se contraste la teoría con la práctica son factores que han llevado a algunos fracasos en la implementación de la gamificación en contextos educativos. Por ello, es importante para realizar una implementación exitosa que el diseño gamificado contribuya a: promover la motivación, permitir la elección y la autonomía, promover la colaboración, la interacción social y la competencia, redefinir el fracaso y permitir 'prueba y error', llevar a que el jugador reflexione y experimente las consecuencias de las elecciones, retroalimentar al jugador, agregar elementos atractivos del diseño de juegos a la instrucción, establecer parámetros de competencia equilibrados, proporcionar un espacio seguro para el andamiaje educativo, promover el compromiso, permitir la progresión y el dominio por parte del jugador, alinear con los principios pedagógicos y contribuir en el logro de los objetivos de aprendizaje, plantear problemas a resolver por los jugadores, despertar el sentido de curiosidad, y usar plataformas o herramientas de apoyo.

Ahora bien, dentro de los principales aportes del presente documento se encuentra la identificación y clasificación de los elementos de juego y los aspectos claves a tener en cuenta en un proceso exitoso de gamificación educativa, y su posterior contrastación y validación en un caso real en el programa de Contaduría Pública a Distancia de la uMNG.

\section{Referencias}

Ahmed, A. \& Sutton, M. J. D. (2017). Gamification, serious games, simulations, and immersive learning environments in knowledge management initiatives. World Journal of Science, Technology and Sustainable Development, 14(2/3), 78-83.

Alsawaier, R. S. (2018). The International Journal of Information and Learning Technology The effect of gamification on motivation and engagement. The International Journal of Information and Learning Technology, 35(1), 56-79. 
Andrade, F. R., Mizoguchi, R. \& Isotani, S. (2016). The bright and dark sides of gamification. International Conference on Intelligent Tutoring Systems, Springer International Publishing.

Antin, J. (2009). Motivated by information: information about online collective action as an incentive for participation. In Proceedings of the ACM 2009 International Conference on Supporting Group Work, ACM, Sanibel Island.

Attali, Y. \& Arieli-Attali, A. (2015). Gamification in assessment: do points affect test performance? Computers and Education, 83(1), 57-63.

Azzam, A. M. (2007). Why students drop out. Educational Leadership, 64(7) 91-93

Barab, S., Zuiker, S., Warren, S., Hickey, D., Ingram-Goble, A., Kwon, E. J. \& Herring, S. C. (2007). Situationally embodied curriculum: relating formalisms and contexts. Science Education, 91(5), 750-782.

Becker, K. (2007). Pedagogy in commercial video games. In D. Gibson, A. Clark \& M. Prensky (Eds). Games and Simulations in Online Learning: Research and Development Frameworks (pp. 21-47). Arlington, VA: Information Science Publishing.

Burgess, T. F. (1991). The use of computerized management and business simulation in the United Kingdom, Simulation \& Gaming An Interdisciplinary Journal, 22, 174-195.

Candido, J., Murman, E. \& McManus, H. (2007). Active learning strategies for teaching lean thinking. Proceedings of the 3rd International CDIO Conference, Cambridge, MA.

Casandra, N. (2017).Technology and Sustainable Development. World Journal of Science, $143(2), 155-171$.

Contreras, R. (2016). Juegos digitales y gamificación aplicados en el ámbito de la educación. RIED. Revista Iberoamericana de Educación a Distancia, 19(2), 27-33.

Craven, D. (2015). Gamification in virtual worlds for learning: a case study of PIERSiM for business. In T.Reiners \& L. C. Wood (Eds). Gamification in Education and Business (pp. 385-401). Cham: Springer.

Dale, S. (2014). Gamification: making work fun, or making fun of work? Business Information Review, 31(2), 82-90.

Deif, A. (2017). International Journal of Lean Six Sigma Insights on lean gamification for higher education. International Journal of Lean Six Sigma, 8(3), 359-376.

Faria, A. J. (1998). Business simulation games: current usage levels: an update. Simulation $\mathcal{E}$ Gaming: An Interdisciplinary Journal, 29, 295-308.

Faria, A. J. \& Wellington, W. J. (2004). A survey of simulation game users, former users and never users. Simulation \& Gaming: An Interdisciplinary Journal, 35, 178-207. 
Faria, A. J. (1987). A survey of the use of business games in academia and business. Simulation and Games, 18, 207-224.

Featherstone, M. (2017). Using gamification to enhance self-directed, open learning in higher education. Recuperado de http://shura.shu.ac.uk/12638/93/Featherstone\%20Using\%20 Gamification\% 20to\%20Enhance\%20Learning.pdf

Folmar, D. (2015). Game it up: Using Gamification to Incentivize your Library. Lanham, MD: Rowman \& Littlefield,

Gallego, C. \& De Pablos, C. (2013). La gamificación y el enriquecimiento de las prácticas de innovación en la empresa: Un análisis de experiencias. Intangible Capital, 9(3), 800-822.

Gåsland, M. (2011). Game mechanic based e-learning. (Master thesis, Norwegian University of Science and Technology).

Gee, J. P. (2005). Learning by design: good video games as learning machines. E-Learning and Digital Media, 2(1), 5-16.

Gee, J. P. (2007). What Video Games Have to Teach us About Learning and Literacy. New York, NY: Palgrave Macmillan.

Gee, J. P. (2014). Games, passion and 'higher' education. In W.G. Tierney, Z. B. Corwin, , T. Fullerton \& G. Ragusa (Eds). Postsecondary Play: The Role of Games and Social Media in Higher Education (pp. 171-189). Baltimore: John Hopkins University Press.

Gibson, D. \& Jakl, P. (2015). Theoretical considerations for game-based e-learning analytics. In T. Reiners \& L.C. Wood(Eds). Gamification in Education and Business (pp. 403-416). Cham: Springer.

Hamari, J. K., Koivisto, J. \& Sarsa, H. (2014). Does gamification work? A literature review of empirical studies on gamification. Proceedings of the 47th Hawaii International Conference on System Sciences, Hawaii, January

Hanus, M. D. \& Fox, J. (2015). Assessing the effects of gamification in the classroom: a longitudinal study on intrinsic motivation, social comparison, satisfaction, effort, and academic performance. Computers E Education, 80(1), 152-161.

Harman, K., Koohang, A., Paliszkiewicz, J. \& Dickinson, P. S. (2014). Scholarly interest in gamification: a citation network analysis Scholarly interest in gamification: a citation network analysis. Industrial Management \& Data Systems, 114(1149), 1438-1452.

Herzig, P., Ameling, M., Wolf, B. \& Schill, A. (2015). Implementing gamification: requirements and gamification platforms. In T. Reiners \& L. C. Wood (Eds). Gamification in Education and Business (pp. 431-471). Cham: Springer. 
Hsu, C. Y., Tsai, C. C. \& Liang, J. C. (2011). Facilitating preschoolers' scientific knowledge construction via computer games regarding light and shadow: the effect of the prediction- observation- explanation (POE) strategy. Journal of Science Education Technology, 20(5), 482-493.

Hughes, M. \& Lacy, C. J. (2016). 'The sugar'd game before thee': gamification revisited. Portal: Libraries.

Hunicke, R., Leblanc, M. \& Zubek, R. (2004). MDA: a formal approach to game design and game research. San Jose, CA: AAAI Workshop on Challenges in GAMEAL,

Issacs, S. (2015). The difference between gamification and game-based learning, Edutopia. Recuperado de http://inservice.ascd.org/the-difference-between-gamification-and-game-based-learning/

Kapp, K. M. (2012a), The Gamification of Learning and Instruction: Game-Based Methods and Strategies for Training and Education. San Francisco, CA: Pfeiffer.

Kapp, K. M. (2012b). Games, gamification, and the quest for learner engagement. Training and Development, 66(6), 64-68.

Kavaliova, M., Virjee, F., Maehel, N. \& Kleppe, I. A. (2016). Crowdsourcing innovation and product development: gamification as amotivational driver. Cogent Business \& Management, 3(1). 1-18

Keeler, A. (2014). Beyond the worksheet: playsheets, GBL, and gamification. Edutopia, 1-3. Recuperado de www.edutopia.org/blog/beyond-worksheet-playsheets-gbl-gamification-alice-keele

Kim, Y., Glassman, M. \& Williams, M. S. (2015). Connecting agents: engagement and motivation in online collaboration. Computers in Human Behavior, 49(1), 333-342.

Kingsley, T. L. \& Grabner-Hagen, M. M. (2015). Gamification: questing to integrate content, knowledge, literacy, and 21st-century learning. Journal of Adolescent \& Adult Literacy, 59(1), 51-61.

Koivisto, J. \& Hamari, J. (2014). Demographic differences in perceived benefits from gamification. Computer in Human Behaviour, 35(June), 179-188.

Kolb, L. (2015). Epic fail or win? Gamifying learning in my classroom. Edutopia, 1-5. Recuperado de www.edutopia.org/blog/epic-fail-win-gamifying-learning-liz-kol

Langendahl, P. A., Cook, M. \& Mark-Herbert, C. (2016). Gamification in higher education. Recuperado de http://pub.epsilon.slu.se/13429/7/langendahl_p_a_etal_160602.pdf

Lawley, E. (2012). Games as an alternate lens for design. Interactions, 19(4), 1. 
Leaning, M. (2015). A study of the use of games and gamification to enhance student engagement, experience, and achievement on a theory-based course of an undergraduate media degree. Journal of Media Practice, 16(2), 155-170.

Lee, J. J. \& Hammer, J. (2011). Gamification in education: what, how, why bother? Academic Exchange Quarterly, 15(2), 1-5.

Li, M. \& Tsai, C. (2013). Game-based learning in science education: a review of relevant research. Journal of Science Education Technology, 22, 877-898.

Li, Q. (2010). Digital game building: learning in a participatory culture. Education Research, $52(4), 427-443$.

Lim, C. P., Nonis, D. \& Hedberg, J. (2006). Gaming in a 3D multiuser virtual environment: engaging students in science lessons. Journal of Education Technology, 37(2), 211-231.

M2 Research Gamification in 2012: Market Update, Consumer and Enterprise Market Trends. Recuperado de http://gamingbusinessreview.com/wp-content/uploads/2012/05/ Gamificationin-2012-M2R3.pdf.

McGonigal, J. (2011). Reality is Broken: Why Games Make Us Better and How They Can Change the World. New York, NY: Penguin Books.

Moizer, J. D., Lean, J., Towler, M. \& Abbey, C. (2009). Simulations and games: overcoming barriers to their use in higher education. Active Learning in Higher Education, 10, 207-224.

Moreno, R. \& Mayer, R. (2000). Engaging students in active learning: the case for personalized multimedia messages. Journal of Education Psychology, 92(4), 724-733.

Morschheuser, B., Hamari, J. \& Koivisto, J. (2016). Gamification in crowdsourcing: a review. 49th Annual Hawaii International Conference on SystemSciences (HICSS). Kauai, Hawaii.

Mozier, J. \& Lean, J. (2010). Toward endemic deployment of educational simulation games: a review of progress and future recommendations. Simulation and Games, 41(1), 116-131.

Myers, R. D. \& Reigeluth, C. M. (2016). Chapter 8: designing games for learning (pre-publication final draft). In C. M. Reigeluth, B. J. Beatty \& R. D. Myers(Eds). Instructional-Design Theories and Models, Vol. IV: The Learner-Centered Paradigm of Education, 1st ed., New York: Routledge. Recuperado de http://researchgate.net

Nicholson, S. (2015). A recipe for meaningful gamification. In T. Reiners \& L. C. Wood, (Eds). Gamification in Education and Business (pp. 1-20) Olso: Springer.

Nilsson, E. \& Jakobsson, A. (2011). Simulated sustainable societies: students' reflections on future cities in computer games. Journal of Science Education Technology, 20(1), 33-50. 
Papastergiou, M. (2009). Digital game-based learning in high school computes science education: impact on educational effectiveness and student motivation. Computers $\mathcal{E}$ Education, 52(1), 1-12.

Pappas, C. (2017). What are the most effective uses of gamification in learning? Ebook: How Gamification Reshapes Learning. Recuperado de https://elearningindustry.com/how-gamification- reshapes-learning

Pendry K. C. (2017). Instructional design talks to game design. On the Horizon, 25(4), 253259. https://doi.org/10.1108/OTH-10-2016-0052

Sánchez, F. Gamificación. (2015). Teoría de la Educación. Educación y Cultura en la Sociedad de la Información, 16(2). 13-15.

Seaborn, K. \& Fels, D. (2015). Gamification in theory and action: a survey. International Journal of Human-Computer Studies, 74(1), 14-31.

Shpakova, A., Dörfler, V. \& Macbryde, J. (2016). Gamification and innovation: a mutually beneficial union. Paper presented at the Conference BAM 2016: 30th Annual Conference of the British Academy of Management, Newcastle upon Tyne.

Simões, J., Redondo, R. D. \& Vilas, A. F. (2013). A social gamification framework for a K-6 learning platform. Computers in Human Behavior, 29(2), 345-353.

Spires, H. A., Rowe, J. P., Mott, B. W. \& Lester, J. C. (2011). Problem solving and game-based learning: effects of middle grade students' hypothesis testing strategies on learning outcomes. Journal of Education Computer Research, 44(4), 453-472.

Squire, K. \& Jan, M. (2007). Mad city mystery: developing scientific argumentation skills with a place-based augmented reality game on handheld computers. Journal of Scientific Education Technology, 16(1), 5-29.

Ting, Y. L. (2010). Using mainstream game to teach technology through an interest framework. Education Technology Society, 13(2), 141-152.

Van den Boer, P. (2013). Introduction to gamification. Recuperado de www.linkedin.com/ in/ pietvandenboer/

Werbach, K. (2014). (Re) Defining gamification: a process approach. International Conference on Persuasive Technology, Springer International Publishing.

Werbach, K. \& Hunter, D. (2012). For the Win How Game Thinking Can Revolutionize Your Business. Philadelphia, PA: Wharton Digital Press, The Wharton School, University of Pennsylvania. 
Wolfe, J. (1993). A history of business teaching games in English-speaking and post-socialist countries: the origination and diffusion of a management education and development technology. Simulation E Gaming: An Interdisciplinary Journal, 24, 446-463.

Zeng, Z., Tang, J. \& Wang, T. (2017). International Journal of Crowd Science Motivation mechanism of gamification in crowdsourcing projects For Authors Motivation mechanism of gamification in crowdsourcing projects. International Journal of Crowd Science Journal of Services, 17(17), 71-82.

Zhang, D. \& Clear, T. (2015). Shaping behaviours through space and place in gamified virtual learning environments. In T. Reiners \& L. C. Wood, (Eds.). Gamification in Education and Business (pp. 331-354). Cham: Springer.

Zichermann, G. \& Cunningham, C. (2011). Gamification by Design. Sebastopol, CA: O'Reilly Media.

Zichermann, G. (2010). Fun is the future: mastering gamification. Google Tech Talk, October 26, Recuperado de www.youtube.com/watch?v=6O1gNVeaE4g 